\title{
From Computational Social Choice to Digital Democracy
}

\author{
Markus Brill \\ Research Group Efficient Algorithms \\ Technische Universität Berlin \\ 10587 Berlin, Germany \\ brill@tu-berlin.de
}

\begin{abstract}
Digital Democracy (aka e-democracy or interactive democracy) aims to enhance democratic decisionmaking processes by utilizing digital technology. A common goal of these approaches is to make collective decision-making more engaging, inclusive, and responsive to participants' opinions. For example, online decision-making platforms often provide much more flexibility and interaction possibilities than traditional democratic systems. It is without doubt that the successful design of digital democracy systems presents a multidisciplinary research challenge. I argue that tools and techniques from computational social choice should be employed to aid the design of online decision-making platforms and other digital democracy systems.
\end{abstract}

\section{The Potential of Digital Democracy}

Recent years have witnessed an increasingly intense debate around the potential (and the risks) of the usage of digital tools for democratic decision-making [Contucci et al., 2019; Sgueo, 2020; Bernholz et al., 2021]. A common goal of digital democracy approaches is to utilize modern information technology - in particular, the Internet-in order to enable more interactive decision-making processes. Designing a digital platform for collective decision-making requires a huge amount of design decisions regarding, for example, interaction possibilities, elicitation techniques, and preference aggregation mechanisms. However, most existing designs are rather ad hoc in nature and little attention is devoted to a principled comparison and evaluation of methods.

The study of collective decision-making lies at the heart of social choice theory [Arrow et al., 2002]. I argue that tools and techniques from social choice theory and, in particular, from computational social choice (COMSOC) [Brandt $e t$ $a l ., 2016 \mathrm{~b}]$, an interdisciplinary research area at the intersection of computer science and economics, should be employed to build and to evaluate digital democracy systems. Putting digital democracy on a solid social-choice-theoretic foundation decreases the risk of employing methods with unintended flaws and has the potential to enable fair and participatory collective decision-making processes even for very large groups.

\section{Enabling Democratic Participation at Scale}

In the following, I provide examples of challenges that are encountered when building digital democracy systems, together with pointers to my own work in COMSOC that is relevant for tackling these challenges. ${ }^{1}$ What these examples have in common is their attempt to make sense of large amounts of contributions stemming from a large number of participants.

\subsection{Liquid Democracy}

The paradigm of liquid democracy (aka delegative voting) aims to reconcile the idealistic appeal of direct democracy with the practicality of representative democracy by allowing participants to choose whether they want to vote directly on a particular issue or whether they want to delegate their vote to somebody they trust [Blum and Zuber, 2016; Valsangiacomo, 2021]. Delegations are topic-specific (i.e., voters can specify different delegatees for different issues), transitive (i.e., voting power accumulates along delegation paths), and delegation decisions can be changed at any time in order to hold delegatees accountable. Liquid democracy, which is an integral part of the digital democracy platform LiquidFeedback [Behrens et al., 2014], enables participation at scale by giving participants the opportunity to have their say on all issues, but not requiring them to do so.

Liquid democracy has been studied theoretically, and applied practically, in various ways in recent years [Ford, 2014; Paulin, 2020]. Many variations and extensions of the basic model have been proposed [Gölz et al., 2018; Colley et al., 2020; Kavitha et al., 2021]. My own work in this area explores ways for making liquid democracy more flexible by allowing voters to delegate different parts of their preference ranking to different delegatees [Brill and Talmon, 2018] or to specify ranked lists of delegatees [Brill et al., 2021a].

\subsection{Preference Elicitation \& Aggregation}

Digital technology also enables novel preference elicitation methods. For example, in so-called pairwise wiki surveys [Salganik and Levy, 2015], participants are repeatedly asked to make pairwise comparisons between alternatives. Each participant can answer arbitrarily many pairwise queries, and

\footnotetext{
${ }^{1}$ I apologize for the focus on my own work, which is due to the nature of the Early Career Spotlight track.
} 
the answers are then used to compute a ranking of all proposals. Importantly, participants also have the option to propose new alternatives and thereby enrich the outcome space. In this way, pairwise wiki surveys combine idea crowdsourcing with preference aggregation in an elegant and scalable way.

Eliciting and aggregating pairwise comparisons between alternatives has a long tradition in psychology [Thurstone, 1927], statistics [Kendall and Babington Smith, 1940], and social choice theory [Young, 1986; Laslier, 1997]. My own work on pairwise aggregation focuses mostly on tournament solutions [Brandt et al., 2016a], including some recent work concerning the margin of victory [Brill et al., 2020b; Brill et al., 2021b], but also comprises work on pairwise aggregation functions that take more information into account [Brill and Fischer, 2012; Aziz et al., 2015; Aziz et al., 2018].

\subsection{Proportional Representation}

A defining feature of participatory digital democracy systems is that all participants are allowed-and encouraged-to contribute to the decision-making process. Since each participant can propose their own alternatives if they are not satisfied with the existing ones (see Section 2.2), a potentially very large number of alternatives needs to be considered. As a result, the order in which competing options are presented plays a crucial role [Behrens et al., 2014]. Ranking options solely by popularity, though intuitively appealing, leads to a "tyranny of the majority" and underrepresents minority opinions. In order to prevent this problem, we need to ensure that the order adequately reflects the opinions of the participants.

The search for orderings that are "representative" in this sense leads to challenging algorithmic problems not unlike those underlying the problem of choosing representative committees [Balinski and Young, 1982; Chamberlin and Courant, 1983; Monroe, 1995]. My own work in this area has mostly focused on proportional representation in the context of approval-based multiwinner voting [Aziz et al., 2017; Brill et al., 2017; Brill et al., 2018; Brill et al., 2020a; Sánchez-Fernández et al., 2021]. Several of the methods discussed in these papers are sequential in nature and can be employed to rank-order alternatives in a proportional way [Skowron et al., 2017]. As a consequence, these methods are directly applicable to the digital democracy scenario described above and also to related scenarios such as ranking questions in live Q\&A events [Israel and Brill, 2021].

\section{Conclusion}

While digital democracy tools are currently mainly used for decision-making within progressive political parties [Blum and Zuber, 2016] or in the context of community engagement platforms such as WeGovNow [Boella et al., 2018], it is quite plausible that, in the foreseeable future, these systems (or their updated versions) will be integrated in - or even be the main component of-democratic decision-making processes on a much larger scale. For this reason, I believe that research on digital democracy systems can have a considerable impact on the future of our democracies.

A multidisciplinary research program is necessary for making digital democracy systems secure, equitable, inclusive, user-friendly, and computationally reliable. I have argued that insights and tools from computational social choice are relevant for this important endeavor.

\section{Acknowledgments}

My work is supported by the Deutsche Forschungsgemeinschaft (DFG) under grant BR 4744/2-1. For more details and references to related work, see my article in the Blue Sky Ideas track of AAMAS [Brill, 2018]. I am greatly indebted to all my coauthors. Furthermore, I would like to thank Ulrike Schmidt-Kraepelin for helpful comments and the members of the MD4SG working group on Civic Participation, led by Paul Gölz and Anson Kahng, for insightful discussions.

\section{References}

[Arrow et al., 2002] K. J. Arrow, A. K. Sen, and K. Suzumura, editors. Handbook of Social Choice and Welfare, volume 1. North-Holland, 2002.

[Aziz et al., 2015] H. Aziz, M. Brill, F. Fischer, P. Harrenstein, J. Lang, and H. G. Seedig. Possible and necessary winners of partial tournaments. Journal of Artificial Intelligence Research, 54:493-534, 2015.

[Aziz et al., 2017] H. Aziz, M. Brill, V. Conitzer, E. Elkind, R. Freeman, and T. Walsh. Justified representation in approval-based committee voting. Social Choice and Welfare, 48(2):461-485, 2017.

[Aziz et al., 2018] H. Aziz, F. Brandl, F. Brandt, and M. Brill. On the tradeoff between efficiency and strategyproofness. Games and Economic Behavior, 110:1-18, 2018.

[Balinski and Young, 1982] M. Balinski and H. P. Young. Fair Representation: Meeting the Ideal of One Man, One Vote. Yale University Press, 1982.

[Behrens et al., 2014] J. Behrens, A. Kistner, A. Nitsche, and B. Swierczek. The Principles of LiquidFeedback. 2014.

[Bernholz et al., 2021] L. Bernholz, H. Landemore, and R. Reich, editors. Digital Technology and Democratic Theory. University of Chicago Press, 2021.

[Blum and Zuber, 2016] C. Blum and C. I. Zuber. Liquid democracy: Potentials, problems, and perspectives. Journal of Political Philosophy, 24(2):162-182, 2016.

[Boella et al., 2018] G. Boella, L. Francis, E. Grassi, A. Kistner, A. Nitsche, A. Noskov, L. Sanasi, A. Savoca, C. Schifanella, and I. Tsampoulatidis. WeGovNow: A map based platform to engage the local civic society. In WWW '18: Companion Proceedings of the The Web Conference 2018, pages 1215-1219. IW3C2, 2018.

[Brandt et al., 2016a] F. Brandt, M. Brill, and P. Harrenstein. Tournament solutions. In F. Brandt, V. Conitzer, U. Endriss, J. Lang, and A. D. Procaccia, editors, Handbook of Computational Social Choice, chapter 3. Cambridge University Press, 2016.

[Brandt et al., 2016b] F. Brandt, V. Conitzer, U. Endriss, J. Lang, and A. Procaccia, editors. Handbook of Computational Social Choice. Cambridge University Press, 2016. 
[Brill and Fischer, 2012] M. Brill and F. Fischer. The price of neutrality for the ranked pairs method. In Proceedings of the 26th AAAI Conference on Artificial Intelligence (AAAI), pages 1299-1305. AAAI Press, 2012.

[Brill and Talmon, 2018] M. Brill and N. Talmon. Pairwise liquid democracy. In Proceedings of the 27th International Joint Conference on Artificial Intelligence (IJCAI), pages 137-143. IJCAI, 2018.

[Brill et al., 2017] M. Brill, R. Freeman, S. Janson, and M. Lackner. Phragmén's voting methods and justified representation. In Proceedings of the 31st AAAI Conference on Artificial Intelligence (AAAI), pages 406-413. AAAI Press, 2017.

[Brill et al., 2018] M. Brill, J.-F. Laslier, and P. Skowron. Multiwinner approval rules as apportionment methods. Journal of Theoretical Politics, 30(3):358-382, 2018.

[Brill et al., 2020a] M. Brill, P. Gölz, D. Peters, U. SchmidtKraepelin, and K. Wilker. Approval-based apportionment. In Proceedings of the 34th AAAI Conference on Artificial Intelligence (AAAI), pages 1854-1861. AAAI Press, 2020.

[Brill et al., 2020b] M. Brill, U. Schmidt-Kraepelin, and W. Suksompong. Refining tournament solutions via margin of victory. In Proceedings of the 34th AAAI Conference on Artificial Intelligence (AAAI), pages 1862-1869. AAAI Press, 2020.

[Brill et al., 2021a] M. Brill, T. Delemazure, A.-M. George, M. Lackner, and U. Schmidt-Kraepelin. Liquid democracy with ranked delegations. Working paper, 2021.

[Brill et al., 2021b] M. Brill, U. Schmidt-Kraepelin, and W. Suksompong. Margin of victory in tournaments: Structural and experimental results. In Proceedings of the 35th AAAI Conference on Artificial Intelligence (AAAI), pages 5228-5235. AAAI Press, 2021.

[Brill, 2018] M. Brill. Interactive democracy. In Proceedings of the 17th International Conference on Autonomous Agents and Multiagent Systems (AAMAS) Blue Sky Ideas track, pages 1183-1187. IFAAMAS, 2018.

[Chamberlin and Courant, 1983] J. R. Chamberlin and P. N. Courant. Representative deliberations and representative decisions: Proportional representation and the Borda rule. The American Political Science Review, 77(3):718-733, 1983.

[Colley et al., 2020] R. Colley, U. Grandi, and A. Novaro. Smart voting. In Proceedings of the 29th International Joint Conference on Artificial Intelligence (IJCAI), 2020.

[Contucci et al., 2019] P. Contucci, A. Omicini, D. Pianini, and A. Sîrbu, editors. The Future of Digital Democracy. Springer International Publishing, 2019.

[Ford, 2014] B. Ford. Delegative democracy revisited. Blog post, 2014. Retrieved June 15, 2021, from http://bford. github.io/2014/11/16/deleg.html.
[Gölz et al., 2018] P. Gölz, A. Kahng, S. Mackenzie, and A. Procaccia. The fluid mechanics of liquid democracy. In Proceedings of the 14th International Workshop on Internet and Network Economics (WINE), pages 188-202. Springer, 2018.

[Israel and Brill, 2021] J. Israel and M. Brill. Dynamic proportional rankings. In Proceedings of the 30th International Joint Conference on Artificial Intelligence (IJCAI). IJCAI, 2021.

[Kavitha et al., 2021] T. Kavitha, T. Király, J. Matuschke, I. Schlotter, and U. Schmidt-Kraepelin. Popular branchings and their dual certificates. Mathematical Programming, 2021.

[Kendall and Babington Smith, 1940] M. G. Kendall and B. Babington Smith. On the method of paired comparisons. Biometrika, 31(3/4):324-345, 1940.

[Laslier, 1997] J.-F. Laslier. Tournament Solutions and Majority Voting. Springer-Verlag, 1997.

[Monroe, 1995] B. L. Monroe. Fully proportional representation. The American Political Science Review, 89(4):925940, 1995.

[Paulin, 2020] A. Paulin. An overview of ten years of liquid democracy research. In Proceedings of the 21st Annual International Conference on Digital Government Research (dg.o), pages 116-121. ACM Press, 2020.

[Salganik and Levy, 2015] M. J. Salganik and K. E. C. Levy. Wiki surveys: Open and quantifiable social data collection. PLoS ONE, 10(5):1-17, 2015.

[Sánchez-Fernández et al., 2021] L. Sánchez-Fernández, N. Fernández, J. A. Fisteus, and M. Brill. The maximin support method: An extension of the D'Hondt method to approval-based multiwinner elections. In Proceedings of the 35th AAAI Conference on Artificial Intelligence (AAAI), pages 5690-5697. AAAI Press, 2021.

[Sgueo, 2020] G. Sgueo. Digital democracy: Is the future of civic engagement online? Technical Report PE 646.161, European Parliamentary Research Service (EPRS), 2020.

[Skowron et al., 2017] P. Skowron, M. Lackner, M. Brill, D. Peters, and E. Elkind. Proportional rankings. In Proceedings of the 26th International Joint Conference on Artificial Intelligence (IJCAI), pages 409-415. IJCAI, 2017.

[Thurstone, 1927] L. L. Thurstone. A law of comparative judgement. Psychological Review, 34(4):273-286, 1927.

[Valsangiacomo, 2021] C. Valsangiacomo. Political representation in liquid democracy. Frontiers in Political Science, 3:591853, 2021.

[Young, 1986] H. P. Young. Optimal ranking and choice from pairwise comparisons. In B. Grofman and G. Owen, editors, Information Pooling and Group Decision Making, pages 113-122. JAI Press, 1986. 